\title{
Correction: A Mindfulness-Based Intervention for Student Depression, Anxiety, and Stress: Randomized Controlled Trial
}

Paul Ritvo ${ }^{1^{*}}, \mathrm{PhD}$; Farah Ahmad ${ }^{2 *}, \mathrm{MPH}, \mathrm{PhD}, \mathrm{MBBS}$; Christo El Morr ${ }^{2 *}$ PhD; Meysam Pirbaglou ${ }^{1}, \mathrm{PhD}$; Rahim Moineddin $^{3}, \mathrm{PhD} ; \mathrm{MVC}^{\mathrm{T}} \mathrm{Tem}^{4}$

${ }^{1}$ School of Kinesiology and Health Science, Faculty of Health, York University, Toronto, ON, Canada

${ }^{2}$ School of Health Policy and Management, York University, Toronto, ON, Canada

${ }^{3}$ Dalla Lana School of Public Health, University of Toronto, Toronto, ON, Canada

${ }^{4}$ York University, Toronto, ON, Canada

*these authors contributed equally

\section{Corresponding Author:}

Christo El Morr, PhD

School of Health Policy and Management

York University

4700 Keele St,

Toronto, ON, M3J1P3

Canada

Phone: 14167362100

Email: elmorr@yorku.ca

\section{Related Article:}

Correction of: https://mental.jmir.org/2021/1/e23491/

(JMIR Ment Health 2021;8(1):e27160) doi: $\underline{10.2196 / 27160}$

In "A Mindfulness-Based Intervention for Student Depression, Anxiety, and Stress: Randomized Controlled Trial" (JMIR Ment Health 2021;8(1):e23491) the authors noted one error.

This paper was inadvertently published with an equal contribution footnote for the authors Paul Ritvo, Farah Ahmad, Christo El Morr, and the group author MVC Team. This was incorrect, as only the authors Paul Ritvo, Farah Ahmad, and
Christo El Morr contributed equally. The equal contribution footnote for MVC Team has been removed.

The correction will appear in the online version of the paper on the JMIR Publications website on January 25, 2021, together with the publication of this correction notice. Because this was made after submission to PubMed, PubMed Central, and other full-text repositories, the corrected article has also been resubmitted to those repositories.

This is a non-peer-reviewed article. Submitted 13.01.21; accepted 13.01.21; published 25.01.21.

Please cite as:

Ritvo P, Ahmad F, El Morr C, Pirbaglou M, Moineddin R, MVC Team

Correction: A Mindfulness-Based Intervention for Student Depression, Anxiety, and Stress: Randomized Controlled Trial JMIR Ment Health 2021;8(1):e27160

URL: https://mental.jmir.org/2021/1/e27160

doi: $10.2196 / 27160$

PMID: 33493128

CPaul Ritvo, Farah Ahmad, Christo El Morr, Meysam Pirbaglou, Rahim Moineddin, MVC Team. Originally published in JMIR Mental Health (http://mental.jmir.org), 25.01.2021. This is an open-access article distributed under the terms of the Creative Commons Attribution License (https://creativecommons.org/licenses/by/4.0/), which permits unrestricted use, distribution, and reproduction in any medium, provided the original work, first published in JMIR Mental Health, is properly cited. The complete bibliographic information, a link to the original publication on http://mental.jmir.org/, as well as this copyright and license information must be included. 